\title{
The Design of the Security Supervision and Evaluation System of Construction Machines and Tools based on the Development of Microsoft Access
}

\author{
ZHANG Jihan ${ }^{1, a^{*}}$, JIANG Ming ${ }^{2, b^{*}}$, LIU Kai ${ }^{3}$, PENG Fei ${ }^{4}$ and Tang Guangrui ${ }^{5}$ \\ 1,2,3,4,5 China Electric Power Research Institute, Beijing 100055, China \\ a zhangjihanjay@qq.com, *b jiangmings@163.com
}

\begin{abstract}
Keywords: construction machines and tools; security evaluation system; Access; database.
Abstract. Aiming at the fulfilling the requirements of security supervision and evaluation of machines and tools in transmission and transformation projects, China Electric Power Research Institute(CEPRI) develops a set of high efficiency of supervision and evaluation system with Microsoft Access. This article introduces the development principle and process, the structure and the function of the system in detail, which includes two parts database and software. The system has been put in use and achieves the original goals.
\end{abstract}

\section{Introduction}

With the general promotion and the comprehensive application of security supervision and evaluation of main construction machines and tools in the transmission and transformation project, the working mode gradually exposes its drawbacks of the isolated information, cumbersome process and inefficiency, in which we record the machines and tools information and reports with paper documents and deal with them by transfer, audition and rectification between the construction companies, supervision companies, the owner companies and CEPRI. Recently, the rapid development of computer technology has brought new opportunities and methods to change the above working mode. It's an urgent affair that we should use the information technology to build a unified database and develop software.

In a transmission and transformation project, the security supervision and evaluation system, limited by construction site conditions, needs to meet the following:

(1) The system can work offline and demand not too high for computer hardware; (2) The scale of the database should not be large, so as to save and transfer the information conveniently by storage devices;

(3) The system should be mastered and operated easily for the operators from different companies.

(4) The maintainability of software should be good enough to fulfill the changes and new problems from different projects, which actually requires a reasonable system structure.

Microsoft Office Access is a piece of relational database management software and the Chinese version is characterized by a whole finished window interface, with a range of tools to collect, retrieve, display and print data. Meanwhile, Access also has some advantages such as powerful function, Intuitive operation and mastering easily, which are more suitable for the development of small and medium sized database system. Taking all these factors, we believe that Access is a good choice to develop.

\section{System development}

Design principle. We used ERWin to design a Microsoft Access database for security supervision and evaluation system of construction machines and tools.

We developed this system software following the software engineering theory, using UML as system Modeling Language and using Rational Rose as system design tool. Throughout the process, we made full use of object-oriented analysis and design method, and the overall analysis and design based on the class diagram analysis. Thus the system model better satisfies the actual needs, with a clear 
and reasonable hierarchy to ensure that the system module division, the interface design are reasonable and it is convenience to achieve the system functional expansion. We developed this system using C\# language in Microsoft Visual Studio 2005. The running platform for this system is Microsoft .NET Framework 2.0.

Development Process. We used repeated incremental model to develop this construction machines and tools system.

(1) We planned the entire project and divided it into two stages: preliminary planning and phase planning. Ratio of the two-stage development progress in the whole content is roughly 2: 8 .

a. Preliminary planning

In this stage, we aimed at obtaining the actual needs for this system, determining the size of the system and choosing risk management strategy to construct this system. We used rapid prototyping methods to obtain the system requirements, determined the user interface style and the user's system requirements specification (including functional and non-functional requirements) through repeated user trial. We carried the change control into the project development process, and brought the short-term results of system development and initial results into the configuration management. The strict change control could ensure system requirements traceability and project plans controllability.

b. Phase planning

In this stage, we completed system downstream development tasks. At the early stages of each phase, we briefly described of the implementation process of the subsystem, and established detailed work steps, according to the requirements specification and phase planning formed after the initial planning. Then project development group would process the detail designing, code programing, check testing, integration and other development tasks in the work sequence for current phase subsystem. After submitting the phase subsystem, the project user group would try it; Project development group had timely access to the user needs' feedback of the subsystem and then updated the development tasks to develop repeatedly. Meanwhile, we introduced a new subsystem to carry out stages of planning and development, thereby ensuring the successful completion of each subsystem development tasks and fully meeting user needs.

(2) At the beginning of each repetition, the project development team changed and updated the detailed system requirements for current phase according to the feedback from the project management team and user groups' trial on the completed outcomes, then added new contents into the incremental development task. We considered the user needs as the baseline and developed through repeated increment to ensure the smooth implementation of the system

\section{System introduction.}

The security supervision and evaluation system of construction machines and tools mainly includes two parts: system database and system software. The data information of database contains the test report of machines and tools as well as the engineering application machine information; Database is divided into two sub-databases, namely foundation database and project database. The system software is divided into four subsystems by the users, i.e., owner company edition, supervision company edition, construction company edition and CEPRI edition. In addition, report library management system used only by CEPRI, start component and management system of report library used by all users publicly also belong to system software.

Database of machines and tools. Data information used in this project is stored in Access database. The database is logically divided into two sub-databases: foundation database (system database) and project database. Foundation database contains 8 data tables, i.e., machines and tools type table, report type table, report requirements table, system test report table, system report file table, system reporting requirements and contents table, machine feature table and system parameter table. Project database contains 9 data tables, i.e., machine information table, test report table, report requirements and contents table, owner company table, supervision company table, construction company table, user table, construction company state table and line segment table. 
The database contains the information of 22 categories construction machines and tools, such as powered winch, holding pole, shackle and grip clamp. The database also contains 7 kinds of reports, including type test report, factory test report, periodic inspection report, installation acceptance report, sample test report, third-party testing reports and other inspection reports.

\section{System software.}

(1) Software structure

This system software can be divided into 4 subsystems--owner company edition, supervision company edition, construction company edition and the CEPRI edition, also including the system report library administrative system used by CEPRI, the system report library review system used by all company and the start components. The structure of the system software is as shown in Figure 3.1.

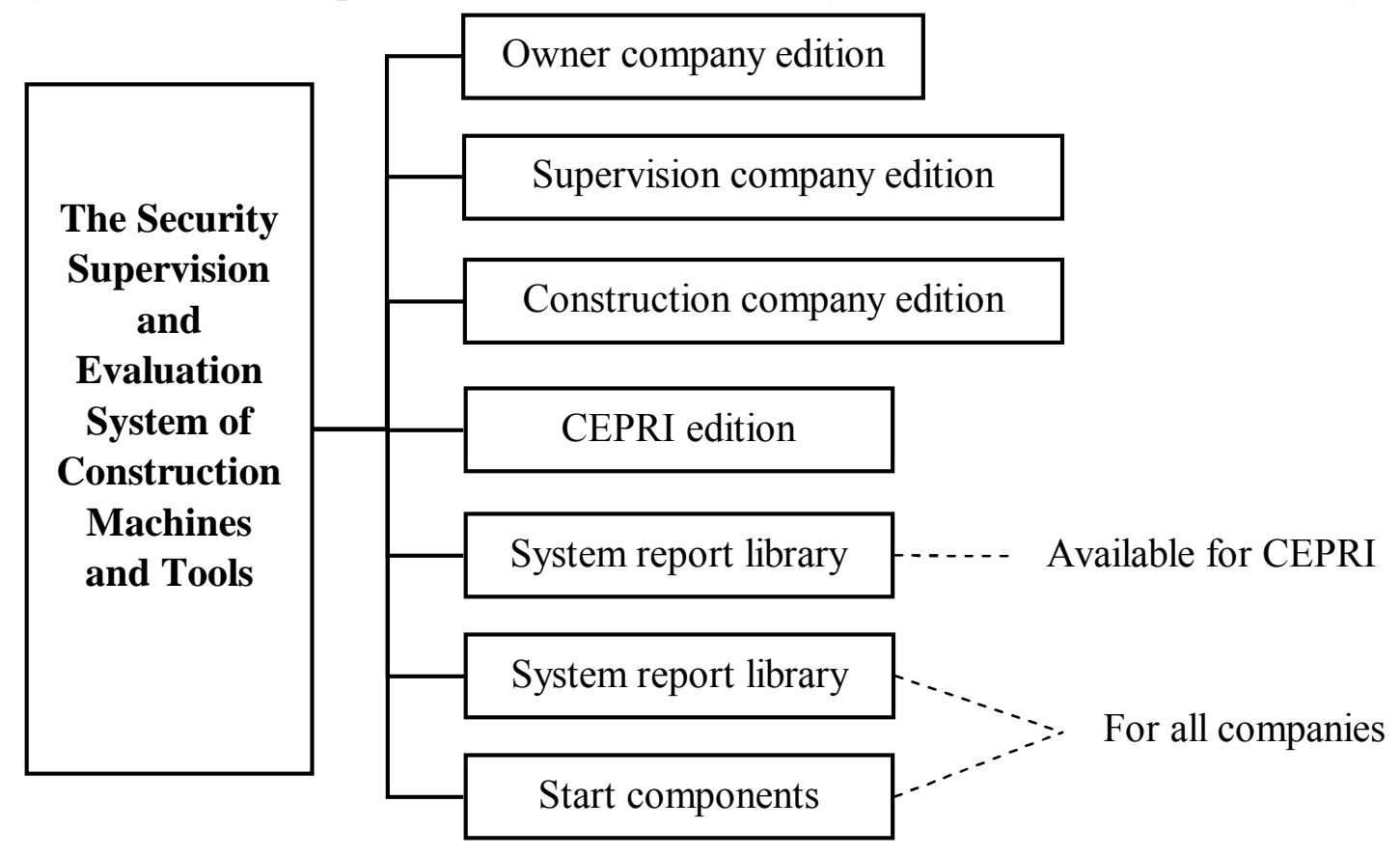

Figure 3.1 system software structure

The owner company edition is used by the owner company of the electric transmission and transformation project for checking the status of the construction company, viewing the information of the construction machines and tools and generating the urging documents. The supervision company edition is used by the supervision company of the electric transmission and transformation project for checking the status of the construction company, viewing the information of the construction machines and tools, generating the review report and information report. The construction company edition is used by the construction company of the electric transmission and transformation project for managing the information of the construction machines and tools and the relative test report, committing the supervision review and generating the rectifying and reforming report. The CEPRI edition is used by the CEPRI for checking the status of the construction company, viewing the information of the construction machines and tools, committing information to the owner company and generating the notification. The system report library administrative system is used for adding, modifying, deleting, querying and reviewing the system report library. The system report library review system is used for querying and reviewing the system report library. The start components are used for providing a unified start entry and GUI for other subsystems except the system report library administrative system, implementing the login control and the invoking of other subsystems.

(2) The main function

The system includes start component, machines and tools category management, construction company management, machines and tools information management, testing reports management, machines and tools information browsing inquiry, machines and tools information audit, generate 
interactive data, system reports management and system reports browse inquiry, 10 functional modules in all. Function of each module is described below.

\section{a. Start components}

Start component realizes the function of calling subsystem and login controlling. Calling subsystem interface includes "owner company", "supervision company", "construction company", "China Electric Power Research Institute(CEPRI)", "program plan" and "System Reports Library" six options, click on any of the options you can call relevant subsystem or open the program file. Login dialog includes selecting a line segment (only required when first logins), selecting the company name (only required when first logins), entering the password. If the password is correct, the system will record the line segment and the company name and login information system, otherwise it will show a prompt.

b. Machines and tools category management

This module lists the categories and the groups of the machines and tools used in the system, and also chooses whether to use as well as evaluate for each group. When selecting a category, the information of the machines and tools will be shown below.

c. Construction company management

According to the categories of the machine and tools, this module lists the construction companies of the system management respectively and audit status of the machines and tools information. When selecting a construction company, its audit data can be imported and checked. When importing the data, the file name of the audit data should be given, and a hint will be shown if the file name chosen by the user is wrong.

d. Machines and tools information management

The information of the machines and tools will be shown in the list in this module; the operations including addition, edit, deleting and etc. can be done. When users add or edit the construction machines and tools information, the field about machines and tools name, product features, manufacturer, specifications model and etc. can be chosen in the list or filled artificially. After users choose a field, detailed instructions for filling will be given.

e. Testing reports management

For a kind of machines and tools information with its testing reports listed, calling, uploading, checking and editing the testing reports can be operated. When users call the testing reports, according to the manufacturer, specifications model and reports type, the system can locate to the corresponding report and detailed information of the reports will be shown. While the users are uploading the testing reports, according to the machines and tools name (groups) and standards of the reports requirements and basis shown in the report type, the system will check the requirements with '*' and require the report content must be qualified and the report file uploaded whose size can't be over 5M.

f. Machines and tools information browsing inquiry

This module can show the list of the machines and tools information; the information can be queried according to filed about the machines and tools name, serial number, product features, specifications model, manufacturer, status and etc..

g. Machines and tools information audit

To audit a piece of machines and tools information, it can be marked by 'Passed' or 'Rectification'; for the information needed to be rectified, the rectification requirement can be written.

h. Generate interactive data

To the owner company, it can generate the rectification urge bill; to CEPRI, it can generate the notification and submit the audit data to the owner company; to the supervision company, it can generate the audit report and data bill, also can submit the audit data to the owner company; to the construction company, it can generate the rectification report and submit the audit data to the supervision company for auditing.

i. System reports management

For the information in the system reports, the operations such as importing, addition, edit, deleting can be carried out.

j. System reports browse inquiry 
Users Choose the report type and the machines and tools type, and then examine the reports information below; the report information can be inquired according to the field about manufacturer, specifications model, report number, test client company, test executing company, number of tests, report date, report validity and etc...

\section{Conclusion}

The security supervision and evaluation system of construction machines and tools has already entered entirely trial operation stage in CEPRI. After more than 20 days of commissioning and maintenance modification, the function of software has already satisfied the requirement of preliminary release. The whole running of the software is stable and the original design goals are basically realized:

1. The storage structure of database is reasonable and no data redundancy exists.

2. The data access during the running of the system is stable and reliable.

3. The function of the system software can satisfy the demand of the users.

4. The software can satisfy the requirement of the system running on usability, security and reliability.

5. Timely and effective technical support and system maintenance are provided during the development, pilot run and operation process of the control system.

The system has been applied to "Lingzhou-Shaoxing $\pm 800 \mathrm{kV}$ HVDC transmission line project", which involves more than 30 construction companies, supervision companies and owner companies in total. During the checking process, the function and performance of the system can entirely meet the requirements of the supervising and checking of the performance of the construction machines and tools.

We believe that this system can make the security supervision and evaluation of the construction machines and tools more systematic and normative, the management of the construction machines and tools information and reports more rational and efficient.

\section{References}

[1] ZhuWenchao, LiuHuizhen, ChenJinazhong. Development of a special-Purpose Database Management system Based on Microsoft Access Client/Server Mode [J]. Journal of Beijing Polytechnic University. 1999.25(2): 114-118

[2] Liu Fang, Xu Yajun, Liang Na. An Application of UML and Modeling Tool Rational Rose [J]. Application Research of Computers, 2002, 19(11):119-120 